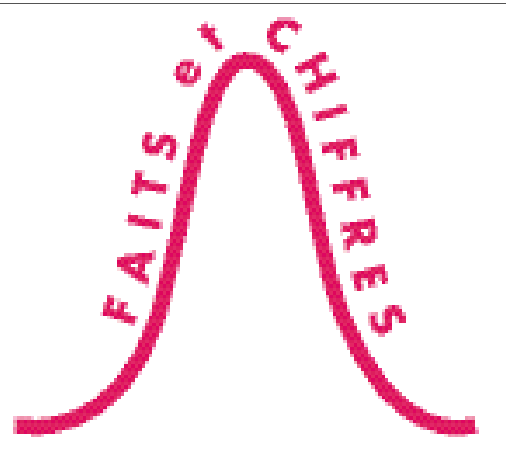

médecine/sciences $1996 ; 12: 677-80$

\title{
Retraite et longévité
}

\section{RÉFÉRENCES}

1. Parant A. Croissance démographique et vieillissement. Population. INED, 1992 ; $\mathrm{n}^{\circ} 6: 1657-76$.

2. Meslé F. La mortalité en France: le recul se poursuit. Population. INED, 1995 ; $n^{\circ} 3: 745-78$.

3. Bourgoin N, Nizard A. La survie des personnes âgées. Population et Sociétés. INED, $1995 ; \mathrm{n}^{\circ} 302$.

4. Actifs ou inactifs ? Situation des assurés à la demande de retraite. Résultats d'une enquête menée en 1993. Retraite et Société. CNAV, $1994 ; n^{\circ} 7$ : 29-47.

5. Lévy ML. Retraites d'aujourd'hui et de demain. Population et Sociétés. INED, 1986 ; $\mathrm{n}^{\circ} 202$.

6. Lévy ML. Quelles institutions pour la Sécurité sociale ? Population et Sociétés. INED, $1987 ; \mathrm{n}^{\circ} 219$.

7. INED. Dix-septième rapport sur la situation démographique de la France, 1988.

8. Commissariat général du Plan. Vieillir demain. Documentation française, 1980.

9. Calot G, Febvay M. La mortalité différentielle suivant le milieu social. Présentation d'une méthode expérimentée en France sur la période 1955-1960. Etudes et conjoncture. INSEE, $\mathrm{n}^{\circ} 11$, novembre 1965.

10. Desplanques G. L'inégalité sociale devant la mort. Données sociales. INSEE, 1993 : 251-8.

D'après Populations et Sociétés.

$\mathrm{m} / \mathrm{s} n^{\circ} 5$, vol. 12, mai 96
D epuis un siècle, la durée de vie moyenne en France a progressé d'environ une année tous les trois ans : de quelque 41 ans à près de 74 ans pour les hommes et d'environ 44 ans à 82 ans pour les femmes. La baisse de la mortalité, très importante et très précoce pour les tout premiers âges de la vie, n'a guère commencé aux âges adultes qu'à partir du début du $\mathrm{XX}^{\mathrm{e}}$ siècle et de façon moins prononcée pour les hommes que pour les femmes, en raison de morts violentes plus fréquentes [1]. Aux âges élevés, elle s'est amorcée encore plus tardivement: un peu avant la Seconde Guerre mondiale pour les femmes, après celle-ci pour les hommes [2, 3]. Comme la mortalité aux jeunes âges est désormais proche de l'incompressibilité, la contribution des âges élevés à l'allongement de la durée de vie moyenne est devenue primordiale, alors qu'elle était négligeable avant guerre. De 1930 à 1990, l'espérance de vie a déjà progressé plus vite, en termes relatifs, à 60 ans qu'à la naissance, plus particulièrement chez les femmes. Cet heureux progrès qu'est la prolongation de la vie humaine fait aujourd'hui problème. Se posent non seulement la question du sens et des conditions de vie des années supplémentaires conquises sur la mort, mais aussi celle des effets combinés des gains de durée de vie et d'une sortie d'activité professionnelle de plus en plus précoce.

\section{Un horizon qui s'éloigne}

En 1960, année où fut instituée la Commission d' études des problèmes de la vieillesse (« Commission Laroque »), celui qui obtenait la liquidation de sa pension avait, le plus souvent, 65 ans* , âge auquel l'espérance de vie était de 12,5 ans pour les hommes, 15,5 ans pour les femmes. Aujourd'hui, si les hommes faisaient toujours valoir à 65 ans leurs droits à pension, ils pourraient escompter percevoir cette dernière en moyenne pendant 16 ans, les femmes un peu plus de 20 ans. Ainsi, à trente ans d'intervalle, si la législation était restée inchangée, le seul calendrier de la mortalité aurait allongé de $25 \%$ et $28 \%$ respectivement, l'horizon temporel des hommes et des femmes prenant leur retraite. Celui-ci s'est, en fait, beaucoup plus éloigné - $55 \%$ pour les hommes et $60 \%$ pour les femmes parce que, depuis le début des années 1970, de nombreuses dispositions ont abaissé à quelque 60 ans l'âge de la retraite, la loi mise en application le $1^{\text {er }}$ avril 1983, ne faisant, comme bien souvent, qu'accom-

\footnotetext{
* Au recensement de 1962, le taux d'activité masculin était de 67,9\% à 60-64 ans et encore de 36,5\% à 65-69 ans. Chez les femmes, proportionnellement moins nombreuses à travailler, à tout âge, que les hommes, le taux d'activité était respectivement égal à $31,9 \%$ et $16,9 \%$.
} 
pagner le mouvement ${ }^{*}$. Ce moindre étalement de l'âge de la fin de l'activité professionnelle - qui est allé de pair avec une entrée et une insertion plus tardives des jeunes sur le marché du travail - est désormais tel qu'apprécier l'évolution du rapport du nombre des retraités aux cotisants à l'aune de celle du rapport démographique des 20-59 ans aux 60 ans ou plus est une approximation de plus en plus satisfaisante.

Dans les conditions anticipées par les projections de l'INSEE et si l'âge de la retraite reste inchangé, la durée de perception de celle-ci s'accroîtrait de près de $38 \%$ pour les hommes et de plus de $31 \%$ pour les femmes de 1990 à 2050. Sur la même période, le flux annuel de nouveaux retraités (les personnes atteignant leur soixantième anniversaire), égal à 550 000-600 000 jusqu'en 2005, devrait ensuite s'élever brutalement, en relation avec l'arrivée des premières générations du «baby-boom», au niveau de 800000 (soit une croissance de quelque $40 \%$ ), pour se stabiliser, à partir de 2030, aux environ de 750000 . Le nombre de personnes atteignant chaque année 60 ans est désormais voisin du nombre de naissances 60 ans plus tôt, la mortalité entre 0 et 60 ans étant devenue faible $(18 \%$ pour les hommes, $8 \%$ pour les femmes dans la table de mortalité 1990-1992) et partiellement compensée statistiquement par l'immigration.

Quant à la proportion de personnes titulaires de pensions complètes pour avoir cotisé le nombre d'années requis, elle devrait, elle aussi, augmenter, du fait de la participation accrue des femmes au monde du travail. Parce qu'elles sont de plus en plus nombreuses à abonder le système, en acquittant des cotisations, les femmes seront logiquement plus nombreuses à tirer des traites sur lui.

\footnotetext{
* Au demeurant, l'âge de la retraite est de plus en plus dissocié de l'âge de cessation d'activité professionnelle. Une enquête récente de la Caisse nationale d'assurance vieillesse (CNAV) a montré que deux futurs retraités sur trois étaient déjà inactifs : un sur quatre était au chômage (ou en préretraite ou en garantie de ressources), un sur quatre était sans activité et un sur dix était invalide, malade
}

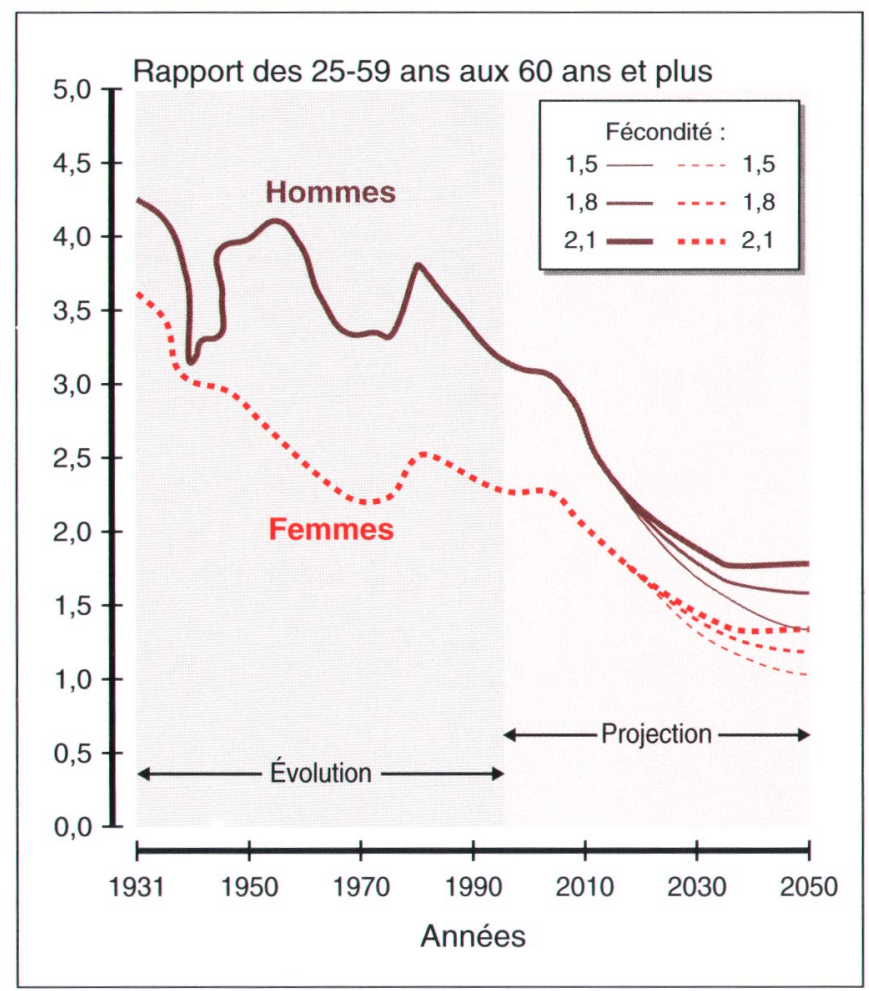

Figure 1. Évolution du rapport des 20-59 ans aux 60 ans et plus. Ce rapport pourrait être diminué de moitié en 2050, abaissé à 1,3-1,8 pour les hommes et 1-1,3 pour les femmes. La signification en est que la charge de 10 retraités incomberait à 11-15 adultes en 2050 contre 27 aujourd'hui.

\section{Trois voies de réforme}

Déjà très important, le besoin de financement du système français des pensions et des retraites est donc inéluctablement appelé à croître. Même dans l'hypothèse d'une croissance vigoureuse et pérenne du revenu national, des réformes s'imposent, d'autant plus urgentes que le temps de réaction de tout système de retraites est fort lent, mais d'autant plus délicates que rien ne peut être brutalement mis en cause. Dans le système instauré au lendemain de la Seconde Guerre mondiale, dit par répartition, le revenu des retraités trouve son origine dans un transfert immédiat entre les générations. Un système fonctionnant suivant le principe de la capitalisation ne permettrait pas d'échapper à la contrainte démographique d'accroissement de la population inactive : les revenus des inactifs sont toujours financés par un prélèvement opéré sur la richesse créée, une année donnée, par la population active occupée [5].

On peut limiter l'ampleur des besoins de financement futurs du système en abaissant le niveau des pensions servies et en les réévaluant de façon moins favorable que par le passé. C'est une des voies empruntées par la réforme des retraites du régime général décidée en juilletaoût 1993. Elle ne peut pas être indéfiniment prolongée. Il serait sans doute inéquitable que le niveau de vie des retraités augmente désormais plus vite que celui des actifs, notamment ceux chargés de famille, alors que la parité est atteinte avec ces derniers. Mais il serait tout autant inéquitable et difficilement accepté par des retraités appelés à peser de plus en plus lourd sur l'échiquier politique, que l'évolution de leur niveau de vie ne soit pas, à l'avenir, sensiblement parallèle à celle du niveau de vie des actifs. 
On peut chercher aussi à accroître les recettes du système selon des modalités moins pénalisantes pour l'emploi que les actuelles cotisations. Cette solution, préconisée dès 1987 par le Comité des Sages constitué pour « animer les États généraux de la Sécurité sociale et garantir leur objectivité »[6] se heurte depuis lors à de graves difficultés politiques. On se bornera ici à examiner comment on s'affranchirait de la contrainte démographique d'accroissement de la population inactive en changeant l'âge du départ en retraite, abstraction faite de l'évolution du chômage.

Le rapport des 20-59 ans aux 60 ans ou plus, qui vaut 2,7 en 1995, pourrait être approximativement divisé par deux d'ici à 2050 et s'abaisser jusqu'à des niveaux compris entre 1,3 et 1,8 pour les hommes, 1 et 1,3 pour les femmes (figure 1). En d'autres termes, en 2050, la «charge » de dix personnes âgées pourrait être répartie entre seulement 11 et 15 adultes, supposés ici tous actifs, quand elle l'était encore entre 27 en 1995.

Circonscrire l'augmentation de l'effort supporté par les actifs en fixant de manière immuable le rap-
Tableau I

ESPÉRANCE DE VIE (EN ANNÉES) DES HOMMES A 60 ANS SELON LA CATÉGORIE SOCIO-PROFESSIONNELLE. ÉVOLUTION DE 1960-1969 À 1980-1989

\begin{tabular}{|lrrr|} 
& $\begin{array}{c}1960 \\
\text { à }\end{array}$ & $\begin{array}{c}1980 \\
\text { à }\end{array}$ & $\begin{array}{r}\text { Gain } \\
\text { (19) }\end{array}$ \\
\hline Professeurs, professions scientifiques et littéraires & 20,8 & 22,2 & 6,7 \\
Instituteurs & 20,5 & 21,4 & 4,4 \\
Cadres administratifs supérieurs & 19,8 & 21,5 & 8,6 \\
Professions libérales et cadres supérieurs & 19,4 & 21,7 & 11,9 \\
Ingénieurs & 19,2 & 22,3 & 16,1 \\
Techniciens & 18,4 & 20,6 & 12,0 \\
Cadres moyens & 18,3 & 20,7 & 13,1 \\
Contremaîtres & 18,2 & 21,2 & 16,5 \\
Cadres administratifs moyens & 18,2 & 20,6 & 13,2 \\
Professions libérales & 18,1 & 21,4 & 18,2 \\
Industriels & 17,9 & 19,0 & 6,1 \\
Agriculteurs exploitants & 17,7 & 20,2 & 14,1 \\
Employés de bureau & 17,3 & 18,6 & 7,5 \\
Patrons de l'industrie et du commerce & 17,4 & 19,5 & 12,1 \\
Artisans & 17,3 & 20,1 & 16,2 \\
Petits commerçants & 17,3 & 19,3 & 11,6 \\
Ouvriers qualifiés & 16,5 & 18,8 & 13,9 \\
Ouvriers & 16,2 & 18,6 & 14,8 \\
Ouvriers spécialisés & 15,9 & 18,4 & 15,7 \\
Salariés agricoles & 15,6 & 18,3 & 17,3 \\
Manœuvres & 15,3 & 17,1 & 11,8 \\
Population masculine totale & 15,9 & 17,9 & 12,6 \\
Source : INSEE [9, 10] & & & \\
& & & \\
\hline
\end{tabular}

Figure 2. Évolution de l'âge frontière entre activité et retraite pour maintenir à 2,7 le rapport des actifs aux retraités. Ce chiffre de 2,7 est celui calculé en 1995. L'âge frontière évoluerait peu jusqu'en 2005, puis s'élèverait rapidement jusqu'en 2050 pour atteindre 70 72 ans. Les projections de la mortalité à cette époque montrent que la durée de vie de retraite serait de 16 à 18 ans pour les hommes et de 20 à 22 ans pour les femmes.

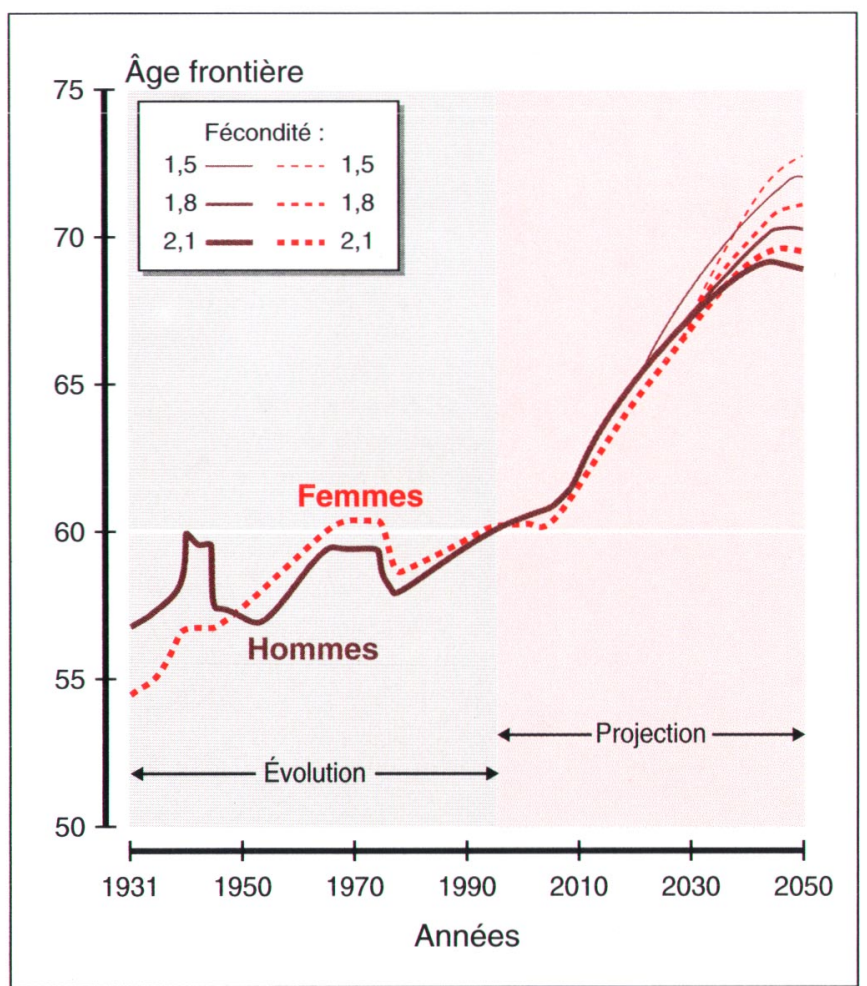


port numérique de ceux-ci aux retraités impliquerait une hausse substantielle de l'âge du départ à la retraite, résultat sur lequel l'INED a déjà attiré l'attention [7]. Si on voulait maintenir le rapport à 2,7 jusqu'en 2050, cet âge frontière augmenterait d'abord lentement - une demie année de 1995 à 2005, de 60 à 60,5 ans - puis beaucoup plus rapidement ensuite - de 9 à 12 ans selon le scénario, en l'espace de 45 ans (figure 2). Prenant alors leur retraite à quelque 70-72 ans, les hommes (nés vers 1980) pourraient encore tabler, avec la mortalité par âge de 2050, sur une vie de retraite de 16 à 18 ans, les femmes de 20 à 22 ans. L'âge auquel l'espérance de vie s'abaisse à une durée déterminée, par exemple 10 ou 20 ans, ne cesse de reculer d'une table de mortalité à la suivante.
Plutôt que d'augmenter de façon identique pour tous l'âge de la retraite, on pourrait aussi imaginer de le moduler pour tenir compte de l'inégalité sociale devant la mort, phénomène, surtout marqué pour le sexe masculin, qui ne s'est guère résorbé depuis sa mise en évidence (Tableau I). C'est ainsi que les membres du groupe "Prospective personnes âgées » du Plan [8] avaient recommandé de ne plus parler d'âge de la retraite, de raisonner exclusivement en termes de durée d'affiliation à un régime et de porter cette dernière à 40 ou 42 , voire 45 ans. Le législateur les a partiellement suivis dans la réforme de juillet-août 1993 des retraites des salariés du secteur privé, allongeant progressivement la durée de cotisation requise mais conservant l'âge de 60 ans pour le bénéfice d'une pen- sion à taux plein. En décembre 1995, les salariés du secteur public et des autres régimes spéciaux ont obtenu que l'allongement de la durée de cotisation ne soit pas étendu à leurs régimes respectifs.

Ainsi, une analyse au premier degré de l'évolution du rapport entre les actifs et les inactifs indique que si la répartition des sacrifices à consentir reste en débat - et en calcul -, leur nécessité ne peut être niée. Il faut pourtant observer que ces considérations ne prennent pas en compte l'augmentation de la productivité du travail des actifs, et donc la question du choix de l'affectation des richesses supplémentaires créées par individu : il s'agit là, en effet, d'un choix fondamental de société que nous n'avions pas pour ambition d'aborder, mais que l'on ne devrait pas éluder en abordant ces questions 